(c) The Author(s), 2021. Published by Cambridge University Press on behalf of The Nutrition Society. This is an Open Access article, distributed under the terms of the Creative Commons Attribution licence (https://creativecommons.org/licenses/by/4.0/), which permits unrestricted reuse, distribution, and reproduction in any medium, provided the original work is properly cited.

\title{
A posteriori dietary patterns in 71-year-old Swedish men and the prevalence of sarcopenia 16 years later
}

\author{
Mikael Karlsson ${ }^{1 *}$, Wulf Becker ${ }^{1}$, Tommy E. Cederholm ${ }^{1}$ and Liisa Byberg ${ }^{2}$ \\ ${ }^{1}$ Department of Public Health and Caring Sciences, Clinical Nutrition and Metabolism, Uppsala University, Uppsala, Sweden \\ ${ }^{2}$ Department of Surgical Sciences, Medical Epidemiology, Uppsala University, Uppsala, Sweden
}

(Submitted 10 March 2021 - Final revision received 7 September 2021 - Accepted 24 September 2021 - First published online 29 September 2021)

\section{Abstract}

The role of diet in sarcopenia is unclear, and results from studies using dietary patterns (DP) are inconsistent. We assessed how adherences to a posteriori DP are associated with the prevalence of sarcopenia and its components 16 years later. Four DP were defined in the Uppsala Longitudinal Study of Adult Men at baseline ( $n$ 1133, average age 71 years). Among 257 men with information at follow-up, 19\% ( $n$ 50) had sarcopenia according to the European Working Group on sarcopenia in Older People 2 definition. Adherence to DP2 (mainly characterised by high intake of vegetables, green salad, fruit, poultry, rice and pasta) was non-linearly associated with sarcopenia; adjusted OR and $95 \% \mathrm{CI}$ for medium and high $v$. low adherence: $0.41(0 \cdot 17,0.98)$ and $0.40(0 \cdot 17,0.94)$. The OR per standard deviation (SD) higher adherence to DP2 was $0 \cdot 70$ $(0.48,1.03)$. Adjusted OR (95\% CI) for 1 sD higher adherence to DP1 (mainly characterised by high consumption of milk and cereals), DP3 (mainly characterised by high consumption of bread, cheese, marmalade, jam and sugar) and DP4 (mainly characterised by high consumption of potatoes, meat and egg and low consumption of fermented milk) were 1.04 (0.74, 1.46), 1.19 (0.71, 2.00) and 1.08 (0.77, 1.53), respectively. There were no clear associations between adherence to the DP and muscle strength, muscle mass, physical performance or sarcopenia using EWGSOP1 (sarcopenia $n$ 54). Our results indicate that diet may be a potentially modifiable risk factor for sarcopenia in old Swedish men.

Key words: Sarcopenia: Dietary pattern: Principal component analysis: Cohort: Longitudinal: Muscle mass

Sarcopenia is recognised as a significant health concern in older individuals ${ }^{(1)}$, including physical impairment, risk of falls and fractures, disability ${ }^{(2)}$, reduced quality of $\operatorname{life}^{(3,4)}$ and higher inhospital and 1-year mortality ${ }^{(2)}$. Sarcopenia was initially defined as an age-related decline in muscle mass. Later, muscle function has been included in most sarcopenia definitions ${ }^{(5-9)}$, since muscle strength, muscle power and physical performance are observed as stronger predictors of clinically relevant outcomes, e.g. functional status, falls and mortality ${ }^{(10-13)}$. Even if it may arise in mid-life, it arises generally as an age-related progression ${ }^{(14)}$.

Since sarcopenia influences the individual's health and wellbeing, it is of great importance to identify contributing modifiable causes. Most studies have focused on the components in the definition as an outcome, rather than sarcopenia itself. Increased physical activity has been reported to have positive effects on muscle mass and improves overall physical function ${ }^{(15,16)}$. However, the effect of diet is less clear, and data are mostly limited to cross-sectional studies ${ }^{(17,18)}$. Studies examining the role of diet in sarcopenia have predominantly focused on single nutrients or food items ${ }^{(18)}$. However, this might not be the best approach when examining effects on chronic diseases in the non-deficient state $^{(19,20)}$. From a public health perspective, it is difficult to provide dietary advice based solely on nutrients, as the nutrient intake is associated with dietary patterns (DP) ${ }^{(21)}$. It is therefore difficult to examine effects of single nutrients or food items without potential influences of both foods and $\mathrm{DP}^{(22)}$. Cross-sectional and longitudinal studies suggest that so-called healthy DP contribute to maintain physical performance in ageing. However, there is limited evidence concerning the relation to muscle mass and muscle strength $^{(17)}$.

In general, a higher adherence to predefined 'healthy DP' have been associated with lower prevalence of sarcopenia, reported both by us ${ }^{(23)}$ and others ${ }^{(24-26)}$, although some 'healthy DP' were not associated with sarcopenia in the same study populations ${ }^{(23,24)}$. Established predefined dietary indexes are often based on current knowledge of the association between diet and the outcome. As the knowledge of associations between diet and sarcopenia is limited, a more exploratory technique, such as data driven so-called a posteriori approaches, might be advantageous. Therefore, we assessed the associations between adherences to a posteriori DP, among 71-year-old Swedish men, and the prevalence of sarcopenia, and its components, 16 years later.

Abbreviations: DP, dietary patterns; ULSAM, Uppsala Longitudinal Study of Adult Men.

*Corresponding author: email mikael.karlsson@pubcare.uu.se 


\section{Materials and methods}

\section{Ethical Approval}

This study was conducted according to the guidelines laid down in the Declaration of Helsinki, and all procedures involving human subjects were approved by the Regional Ethical Review Board at Uppsala University; Dnr 251/90 \& 2007/338. Written informed consent was obtained from all subjects.

\section{Study population}

This study is based on the Uppsala Longitudinal Study of Adult Men (ULSAM) ${ }^{(27)}$, an ongoing population-based cohort study that started in 1970 when all men born 1920-1924 and living in Uppsala County were invited to a health examination; 2322 men participated (participation rate $82 \%$ ), outlined in Supplementary Fig. 1. Reexaminations of these men have been carried out at the approximate ages of $60,70,77,82,88$ and 93 years.

Baseline for the present study was the third investigation cycle conducted in 1991-1995 (ULSAM3), at the average age of 71 years, when 1221 men participated (participation rate $73 \%$ ) in the reinvestigation. In order to define the DP, availability of dietary data at baseline ( $n$ 1138) and a reported energy intake between 800 and $4000 \mathrm{kcal} / \mathrm{d}^{(28)}$ ( $n$ 1133) were required. Followup was the sixth investigation cycle conducted in 2008-2009 (ULSAM6), at the average age of 87, when 354 men participated (participation rate $58 \%$ ). Of these 354 men, 39 had missing data on dietary intake in ULSAM 3 and were excluded. The examination at ULSAM6 included physical function tests and 290 of the participants completed body composition measurement using whole-body dual-energy X-ray absorptiometry, as described below. In total, 315 men were included in the analyses of DP and the constituent variables in the definition of sarcopenia. In 257 men, data were available to define sarcopenia according to the latest EWGSOP definition ${ }^{(29)}$, and none of these participants had missing values in covariates.

Dietary assessment, food grouping and a posteriori dietary pattern

Information on dietary intake was collected during seven consecutive days using a pre-coded menu book (online Supplementary Material 1) from the Swedish National Food Agency, giving preprinted alternatives for food items, dishes and when the meals are consumed. Food intake was reported in household measures or in predefined portion sizes. Consumptions not pre-coded in the menu book were to be reported in free-text ${ }^{(30)}$. A dietician or a trained nurse instructed the participant on how to use the menu book, which also included written instructions and pictures of portions. Dietary data were analysed with commercial software using a food composition database from the Swedish National Food Agency (SLV version 1990). The menu book was validated in a subgroup of the study population ${ }^{(31)}$, by comparison with open-ended weighed food records, displaying a larger proportion of participants under-reporting energy intake compared to weighed food records, but with moderate to high agreements between the methods regarding intake of macro and micro-nutrients.
DP were defined based on the 1133 men (ULSAM3) having a reported dietary intake during $7 \mathrm{~d}$. Mean intake of the seven recoding days of reported food items was categorised into twenty-six food groups, based on the food item's nutrient composition and how the food item is used in cooking or eaten in the population studied (online Supplementary Table 1). For example, milk and fermented milk products were divided into different groups because fermented milk is commonly used together with cereals at breakfast or as a light meal, and milk has been used as a beverage in Sweden since the $1930 \mathrm{~s}^{(32,33)}$. Cold meats include food items often used on bread at breakfast or as light meals. In Sweden, potatoes are used in a similar way to rice and pasta in other countries and are therefore excluded from the food group Vegetables. Tea, coffee and tap water were excluded as they did not significantly contribute neither to energy intake nor to DP separation. Another reason for excluding tap water was due to probable misreporting. Cream (385 men had a reported intake, mean intake $<6 \mathrm{~g} / \mathrm{d}$ ) and vegetable oils (three men had a reported intake, mean intake $<5 \mathrm{~g} / \mathrm{d}$ ) were excluded due to infrequent consumption. Butter and margarine were excluded as they may be misclassified (in Sweden margarine is often called 'butter' in everyday language), and fat used for cooking was included in dishes. The exclusion of these food items rendered a greater proportion of variance explained and more distinct DP, but did not affect the characteristic features of the DP. In order to preserve a wider variation in dietary intake, the dietary intake was not adjusted for the total energy intake when DP were defined ${ }^{(34)}$. However, total energy intake was included in the multivariable adjusted analyses of DP in relation to the outcomes.

DP were defined using principal component analysis, which reduces larger amounts of observed variables to a smaller number of principal components while maximising the variance and identifying structures in the observed data. Bartlett's test of sphericity $(<0.001)$, indicating that the variables are related, and Kaiser-Meyer-Olkin measure of sampling (0.557), indicating the proportion of variances that might be caused by underlying factors, together indicated that the factor analysis was suitable for structure detection and useful with our data. We applied a commonly used threshold ${ }^{(34,35)}$ (principal components loadings $>0.30$ and $<-0.30$ for each food group) for interpretation of the DP (online Supplementary Table 2); however, DP were not labelled on the basis of this interpretation.

The top four principal components were selected based on a combined assessment of a break point (elbow) in the scree plot, eigenvalues ( $>1.5)$ and domestic-cultural knowledge. The identified principal components were rotated with varimax, creating orthogonal (uncorrelated) factors that form the defined DP for this study. The four chosen DP (DP1, DP2, DP3 and DP4) accounted for $28 \%$ of the total variance.

Using the postestimation command predict, each participant was given a factor score, based on the retained factors, to reflect the agreement with each DP. Hence, a high factor score indicates a high intake from food groups that loaded positively and low intake of food groups loading negatively on the given DP. Based on the factor scores' tertile percentiles in the total population of 1133 men at baseline, participants were categorised as having low, medium or high adherence to a given DP. 


\section{Definition of sarcopenia}

The European Working Group on Sarcopenia in Older People (EWGSOP) proposed diagnostic criteria for sarcopenia in $2010^{(36)}$, denoted as EWGSOP1. These criteria were revised in $2019^{(29)}$ and denoted as EWGSOP2. Sarcopenia defined according to EWGSOP2 was the main outcome of this study and defined as the combination of low muscle strength (reduced hand grip strength, $<27 \mathrm{~kg}^{(37)}$ and/or five-times chair stand test, $>15 \mathrm{~s}$ ) ${ }^{(38)}$ and low muscle mass (appendicular lean mass index, lean mass index, $\left.<7.0 \mathrm{~kg} / \mathrm{m}^{2}\right)^{(39)}$. Severity grading of sarcopenia was not done.

Sarcopenia defined according to EWGSOP1 was used in sensitivity analysis, to allow comparison with previous published studies. Participants were categorised as having sarcopenia, i.e. having low muscle mass together with either low hand grip strength and/or low gait speed or otherwise as not having sarcopenia. The chosen cut-off values were for appendicular lean mass index $<7.26 \mathrm{~kg} / \mathrm{m}^{2}$ (low muscle mass) ${ }^{(40)}$, hand grip strength $<30 \mathrm{~kg}$ (low muscle strength) ${ }^{(41)}$ and gait speed $<0.8 \mathrm{~m} / \mathrm{s}$ (low physical performance) ${ }^{(41)}$.

\section{Muscle strength, muscle power and physical performance}

Handgrip strength was measured using an adjustable hydraulic hand dynamometer (Fabrication Enterprises, White Plains), considered to measure grip strength with the same precision as the Jamar hydraulic hand dynamometer ${ }^{(42)}$. Participants were sitting on a chair with the arm supported, shoulder relaxed, elbow at an angle of $90^{\circ}$, the wrist in a neutral position and feet on the floor. Measurement started with the dominant hand and the highest of three results for each hand was recorded, whereof the highest value, regardless if dominant hand or not, was used. In total, 301 participants performed the test at follow-up; two participants were unable to perform the test due to pain or disease and 12 due to unknown reasons.

The chair stand test included five chair rises, as fast as possible in a safe manner, with arms crossed over the chest. The duration was measured, to the nearest $0.5 \mathrm{~s}$, from the first rise until seated ageing after the fifth rise. A total of 241 participants performed five rises, sixty-six participants tried but were unable to perform the test, four participants declined to perform the test or were unable due to medical reasons and four participants had missing value due to unknown reasons. Participants who failed the chair stand test were handled as having a result above the cut-off ( $>15 \mathrm{~s}$ ) in the sarcopenia definition but were excluded in analyses using chair stand test as outcome

A self-chosen comfortable walking speed was measured using the intermediate $6 \mathrm{~m}$ of a distance of $10 \mathrm{~m}$ with no obstacles. Participants were allowed to use walking aid if they preferred to. In total, 257 participants performed the test at follow-up. Reasons for missing data included home visit and therefore not tested ( $n$ 50), unable to perform the test due to disease or lack of strength $(n 7)$ or due to unknown reason $(n 1)$. The fifty-eight participants with missing data were excluded from analyses using gait speed as outcome.

\section{Anthropometry and body composition measurements}

Body height was measured to the nearest centimeter and body weight to the nearest $0 \cdot 1 \mathrm{~kg}$. BMI was calculated as the ratio of the weight to the squared height $\left(\mathrm{kg} / \mathrm{m}^{2}\right)$.

Lean muscle mass was measured using dual-energy X-ray absorptiometry (DPX Prodigy, Lunar corp.). Precision errors of dual-energy X-ray absorptiometry measurements in the present laboratory have been calculated to be $1.5 \%$ for total fat mass and $1.0 \%$ for total lean mass ${ }^{(43)}$. Appendicular lean mass index was calculated as the ratio of appendicular muscle mass (lean mass of legs and arms) to the squared height $\left(\mathrm{kg} / \mathrm{m}^{2}\right)^{(40)}$.

\section{Covariates used for regression analyses}

Questionnaires on education, physical activity and smoking habits were completed at baseline (ULSAM3) under standardised conditions, as earlier described ${ }^{(44)}$. Information on educational level was categorised by years in school (6-7 years, $8-13$ years or $>13$ years). The leisure-time physical activity was defined as sedentary, moderate, regular or athletic ${ }^{(45)}$ using a validated questionnaire ${ }^{(46)}$. Physical activity was then categorised in three subgroups: low (sedentary and moderate), medium (regular) or high (athletic). Smoking status was categorised as never, former or current smoker.

The follow-up period was defined as the period from the date of examination at baseline to the date of dual-energy X-ray absorptiometry measurement at follow-up.

Charlson's unweighted Comorbidity Index ${ }^{(47,48)}$ was calculated based on in-patient diagnoses from patient records recorded before baseline. The comorbidity score was then dichotomised according to score $(0$ and $\geq 1)$.

Age, follow-up period, reported energy intake and BMI were used as continuous variables in statistical analyses. Education, physical activity, smoking and the Charlson Comorbidity Index were used as categorical variables.

\section{Statistical analyses}

The association between each DP and the prevalence of sarcopenia was analysed using logistic regression models estimating OR and their $95 \% \mathrm{CI}$.

Further, we assessed the associations between DP and the four components in the definition of sarcopenia (i.e. handgrip strength, chair stand test, appendicular lean mass index and gait speed) using linear regression analyses estimating beta coefficients and their $95 \% \mathrm{CI}$.

For each of these analyses, linear and non-linear associations were explored. In the linear model, each DP was entered as a standardised continuous variable with mean 0 and 1 SD increments. Non-linear associations were first explored with each DP as a categorical variable, according to low, medium or high adherence, based on their factor scores and tertile limits determined in the total baseline population of 1133 men. A joint Wald test was performed to evaluate whether factor indicators are equal to 0 . Next, each DP was entered as a standardised continuous variable with $1 \mathrm{sD}$ increments using restricted cubic splines with three knots placed at the 10th, 50th and 90th 
percentiles of the DP distribution, as recommended by Harrell ${ }^{(49)}$, and with the median used as reference point, illustrating potential nonlinear associations; results are presented as graphs.

A directed acyclic graph approach was applied using DAGitty ${ }^{(50)}$ (online Supplementary Fig. 2) to identify potential confounders to be included in the multivariable models ${ }^{(51)}$. The directed acyclic graph is a graphical presentation of the assumed causal relationships between factors relevant for the current research question. Based on these assumptions and assuming no residual confounding, DAGitty provides information about minimal sufficient adjustment sets for estimating the total effect of an exposure on the outcome. We applied three models. Model 1 was unadjusted for potential confounders. Model 2 was adjusted for age at baseline, follow-up period, reported energy intake at baseline, education, physical activity level at baseline, smoking and morbidity at baseline. Model 3 was further adjusted for BMI at baseline. Model 3 is in agreement with the minimal sufficient adjustment set suggested by DAGitty.

All analyses were performed in Stata version 15.1 (Stata Corp).

\section{Results}

\section{Characteristics of the studied population}

There were some differences in baseline characteristics between the main study population of 257 men with follow-up information and the total group of 1133 men with dietary information at baseline (online Supplementary Table 3). The group of 257 men had a lower BMI, a higher reported energy intake, a smaller proportion were smokers and had more years of education, higher reported physical activity level and a lower Charlson Comorbidity Index. Differences in average dietary intake were small and not clinically relevant (Table 1 and online Supplementary Table 4).

Characteristics of the 257 men in our main study population at baseline and at follow-up are displayed by DP adherence in Table 2 and in Supplementary Table 5. According to EWGSOP2 (total $n$ 257), 50 (19\%) had confirmed sarcopenia. When using the definition EWGSOP1 (total $n$ 255) 54 (21\%) had confirmed sarcopenia. Thirty-three participants were defined as having sarcopenia by both definitions. Compared with those without sarcopenia, participants with confirmed sarcopenia (EWGSOP2) had a lower body weight and BMI both at baseline $\left(73.3 \mathrm{~kg} v .81 \cdot 0 \mathrm{~kg}, 24 \cdot 1 v \cdot 26 \cdot 3 \mathrm{~kg} / \mathrm{m}^{2}\right)$ and at follow-up (68.6 kg v. $78.1 \mathrm{~kg}, 23.2 v .26 .2 \mathrm{~kg} / \mathrm{m}^{2}$ ). There were no mean differences between participants with or without sarcopenia in terms of reported intake of energy $(\mathrm{kcal} / \mathrm{d})$ or protein $(\mathrm{g} / \mathrm{d})$, years of education, reported physical activity level, prevalence of smoking or comorbidity at baseline.

\section{Dietary patterns}

Factor loadings of food groups in the DP (online Supplementary Table 2) correspond to differences in the mean dietary intake, displayed in Supplementary Table 4 . The four DP explained $28 \%$ of the total variance. DP1 (7.9\% of total variance) was mainly characterised by high positive loadings for milk and cereals, indicating that participants with high adherence to DP1 consumed greater amounts of these foods, and high negative loadings of alcohol. Thus, participants with high adherence to DP1 consumed, on average, more than three times as much milk, but three times less alcohol as compared with those with low adherence. DP2 (6.9\% of total variance) mainly represents a DP with high consumption of vegetables, green salad and fruit as well as poultry, rice and pasta. For example, those with high adherence to DP2 consumed on average more than one fruit ( $180 \mathrm{~g}$ ) per day, and their consumption of fruits and vegetables was three times higher, compared with those with low adherence. DP3 (6.7\% of total variance) was mainly characterised by high consumption of bread, cheese and marmalade, jam and sugar. The intake of these foods among participants with high adherence to DP3 was twice as high compared with those with low adherence. Positive loadings for potato, meat and egg and negative loading for fermented milk mainly characterised DP $4 \quad(6 \cdot 1 \%$ of total variance). Participants with high adherence to DP4 reported a mean intake of $763 \mathrm{~g}$ meat per week, $300 \mathrm{~g}$ more than those with low adherence ( $455 \mathrm{~g}$ ). On average, there were only small differences in consumption of fruit and vegetables regardless of adherence to DP4.

The mean intake of selected nutrients is displayed in Supplementary Table 6 , based on adherence to the respective DP.

\section{Dietary patterns and sarcopenia}

The associations between the DP and sarcopenia (EWGSOP2) are displayed in Table 3 . When the DP were used as continuous variables (estimates expressed per $1 \mathrm{SD}$ increment), DP1, DP3 and DP4 had an adjusted OR (model 3) above 1.0 (1.04, 1.19 and $1 \cdot 08$, respectively), with wide CIs. The adjusted OR (model 3) for DP2 and sarcopenia was 0.70 (95\% CI: 0.48, 1.03).

In analyses with the DP as categorical variables medium and high adherence $v$. low adherence; Table 3), the p-values from the Wald tests were 0.91, 0.06, 0.40 and 0.47 for DP1, DP2, DP3 and DP4, respectively, indicating that the association between DP2 and sarcopenia may be nonlinear. Compared with low adherence to DP2, both medium and high adherence was associated with lower OR of sarcopenia, adjusted OR (model 3): 0.41 (95\% CI: 0.17, 0.98) and 0.40 (95\% CI: $0 \cdot 17,0.94)$, respectively. No clear patterns were observed for DP1, DP3 or DP4 in relation to sarcopenia and confidence intervals were wide (Table 3, online Supplementary Fig. 3). Discrepancies between linear and categorical OR for DP3, where the linear OR was 1.19/SD (95 \% CI: $0 \cdot 71,2 \cdot 00)$ and medium and high adherence $(v$. low adherence) had adjusted OR of 0.50 (95\% CI: 0.18, 1.40) and 0.53 (95\% CI: $0 \cdot 17,1 \cdot 70)$ can be explained by the cut-off limits indicated in Supplementary Fig. 3. A higher adherence to DP4 was associated with higher odds ratio of sarcopenia although CI were wide (OR: 1·61, $95 \%$ CI: 0·67, 3.87, model 3).

No clear associations were observed between DP and sarcopenia, defined according to EWGSOP1 (online Supplementary Table 7). 
Table 1. Dietary intake among men included in the main analysis, displayed as food groups at baseline by adherence to each dietary pattern at baseline (Mean values and standard deviations).

\begin{tabular}{|c|c|c|c|c|c|c|c|c|c|c|c|c|c|c|c|c|c|c|c|c|c|c|c|c|c|c|}
\hline & \multirow{2}{*}{\multicolumn{2}{|c|}{ Total }} & \multicolumn{6}{|c|}{ DP 1} & \multicolumn{6}{|c|}{ DP 2} & \multicolumn{6}{|c|}{ DP 3} & \multicolumn{6}{|c|}{ DP 4} \\
\hline & & & \multicolumn{2}{|c|}{ Low } & \multicolumn{2}{|c|}{ Medium } & \multicolumn{2}{|c|}{ High } & \multicolumn{2}{|c|}{ Low } & \multicolumn{2}{|c|}{ Medium } & \multicolumn{2}{|c|}{ High } & \multicolumn{2}{|c|}{ Low } & \multicolumn{2}{|c|}{ Medium } & \multicolumn{2}{|c|}{ High } & \multicolumn{2}{|c|}{ Low } & \multicolumn{2}{|c|}{ Medium } & \multicolumn{2}{|c|}{ High } \\
\hline & Mean & SD & Mean & SD & Mean & SD & Mean & SD & Mean & SD & Mean & SD & Mean & SD & Mean & SD & Mean & SD & Mean & SD & Mean & SD & Mean & SD & Mean & SD \\
\hline Food groups* & $n 257$ & & $n 98$ & & $n 68$ & & $n 91$ & & $n 74$ & & $n 91$ & & $n 92$ & & $n 67$ & & $n 81$ & & $n 109$ & & $n 100$ & & $n 83$ & & $n 74$ & \\
\hline Cheese & 35 & 21 & 35 & 20 & 40 & 24 & 32 & 20 & 32 & 20 & 33 & 20 & 40 & 22 & $21 \dagger$ & $14 \dagger$ & $29 \dagger$ & $15 \dagger$ & $48 \dagger$ & $21 \dagger$ & 34 & 18 & 35 & 23 & 36 & 23 \\
\hline Bread & 108 & 41 & 107 & 34 & 115 & 48 & 104 & 43 & 101 & 39 & 104 & 36 & 119 & 46 & $73+$ & $19 \dagger$ & $101 \dagger$ & $33+$ & $136 \dagger$ & $38 \dagger$ & 99 & 32 & 107 & 43 & 122 & 47 \\
\hline Fermented milk & 99 & 102 & 131 & 102 & 108 & 108 & 58 & 81 & 83 & 98 & 98 & 103 & 113 & 103 & 59 & 71 & 93 & 92 & 129 & 114 & $144 \dagger$ & $108 \dagger$ & $82 \dagger$ & $84 \dagger$ & $59 \dagger$ & $87 \dagger$ \\
\hline Milk & 230 & 188 & $114 \dagger$ & $110 \dagger$ & $206 \dagger$ & $144 \dagger$ & $373 \dagger$ & $191 \dagger$ & 232 & 185 & 226 & 198 & 233 & 182 & 204 & 175 & 242 & 194 & 237 & 192 & 200 & 164 & 235 & 182 & 265 & 219 \\
\hline Soup & 28 & 40 & 27 & 40 & 30 & 40 & 26 & 40 & 23 & 31 & 22 & 30 & 37 & 51 & 25 & 47 & 24 & 36 & 32 & 37 & 35 & 44 & 22 & 31 & 24 & 41 \\
\hline Pancak & 21 & 26 & 11 & 21 & 19 & 19 & 33 & 30 & 27 & 30 & 16 & 21 & 21 & 25 & 15 & 21 & 22 & 23 & 25 & 29 & 26 & 26 & 20 & 27 & 15 & 23 \\
\hline Quiche and pie & 8 & 17 & 8 & 18 & 11 & 20 & 6 & 15 & 11 & 19 & 8 & 18 & 6 & 15 & 5 & 12 & 6 & 15 & 12 & 21 & 12 & 21 & 8 & 17 & 4 & 12 \\
\hline Salad with proteir & 7 & 15 & 14 & 21 & 3 & 8 & 1 & 3 & 2 & 5 & 5 & 13 & 12 & 20 & 6 & 15 & 6 & 16 & 7 & 15 & 7 & 14 & 6 & 15 & 7 & 17 \\
\hline Cold meats & 14 & 14 & 15 & 13 & 18 & 16 & 10 & 12 & 12 & 12 & 14 & 14 & 15 & 15 & 11 & 11 & 13 & 14 & 16 & 15 & 10 & 10 & 13 & 13 & 20 & 17 \\
\hline Meat & 65 & 28 & 61 & 26 & 62 & 27 & 71 & 30 & 66 & 24 & 66 & 26 & 63 & 32 & 63 & 28 & 65 & 27 & 66 & 29 & $50 \dagger$ & $23+$ & $62 \dagger$ & $20 \dagger$ & $88 \dagger$ & $27 \dagger$ \\
\hline Poultry & 5 & 8 & 7 & 9 & 6 & 9 & 2 & 6 & $1 \dagger$ & $2 \dagger$ & $4 \dagger$ & $6 \dagger$ & $10 \dagger$ & $11 \dagger$ & 6 & 10 & 5 & 8 & 5 & 8 & 5 & 8 & 4 & 7 & 6 & 10 \\
\hline Egg & 14 & 17 & 18 & 21 & 13 & 14 & 11 & 14 & 15 & 18 & 14 & 16 & 14 & 18 & 13 & 17 & 13 & 19 & 15 & 16 & $7 \dagger$ & $9 \dagger$ & $13 \dagger$ & $16 \dagger$ & $24 \dagger$ & $22 \dagger$ \\
\hline Seafood & 4 & 8 & 4 & 9 & 4 & 6 & 4 & 8 & 2 & 5 & 5 & 8 & 4 & 8 & 3 & 7 & 4 & 8 & 4 & 7 & 4 & 7 & 3 & 8 & 4 & 8 \\
\hline Vegetable & 26 & 26 & 23 & 21 & 24 & 23 & 29 & 33 & $13 \dagger$ & $11 \dagger$ & $19 \dagger$ & $13 \dagger$ & $42 \dagger$ & $36 \dagger$ & 25 & 31 & 28 & 28 & 24 & 22 & 26 & 27 & 19 & 19 & 32 & 31 \\
\hline Green salad & 34 & 31 & 42 & 35 & 30 & 25 & 29 & 29 & $18 \dagger$ & $18 \dagger$ & $32 \dagger$ & $26 \dagger$ & $49 \dagger$ & $36 \dagger$ & 25 & 23 & 37 & 34 & 38 & 32 & 32 & 31 & 36 & 32 & 36 & 29 \\
\hline Legumes & 16 & 26 & 8 & 17 & 13 & 22 & 28 & 31 & 19 & 29 & 14 & 22 & 17 & 25 & 15 & 25 & 17 & 26 & 17 & 26 & 13 & 21 & 20 & 29 & 18 & 26 \\
\hline Juice & 30 & 58 & 27 & 58 & 39 & 56 & 28 & 59 & 15 & 33 & 17 & 33 & 56 & 80 & 15 & 33 & 29 & 57 & 41 & 67 & 47 & 77 & 19 & 34 & 21 & 43 \\
\hline Fruit & 124 & 101 & 110 & 85 & 121 & 93 & 141 & 119 & $60 \dagger$ & $56 \dagger$ & $119 \dagger$ & $74 \dagger$ & $180 \dagger$ & $118 \dagger$ & 99 & 91 & 126 & 101 & 137 & 104 & 155 & 111 & 110 & 87 & 98 & 90 \\
\hline Pasta and rice & 20 & 26 & 20 & 22 & 21 & 29 & 19 & 29 & $7 \dagger$ & $9 \dagger$ & $16 \dagger$ & $18 \dagger$ & $34 \dagger$ & $34 \dagger$ & 21 & 25 & 19 & 25 & 20 & 29 & 20 & 23 & 14 & 17 & 27 & 36 \\
\hline Potato & 145 & 64 & 127 & 50 & 145 & 51 & 163 & 79 & 140 & 54 & 142 & 57 & 150 & 77 & 125 & 59 & 145 & 53 & 156 & 72 & $117 \dagger$ & $50 \dagger$ & $148 \dagger$ & $48 \dagger$ & $179 \dagger$ & $79 \dagger$ \\
\hline Cereals & 85 & 97 & $23 \dagger$ & $33+$ & $56 \dagger$ & $56 \dagger$ & $174 \dagger$ & $102 \dagger$ & 80 & 88 & 88 & 95 & 86 & 106 & 79 & 100 & 100 & 105 & 78 & 88 & 74 & 83 & 88 & 98 & 97 & 112 \\
\hline Dessert & 92 & 70 & 69 & 52 & 102 & 71 & 108 & 80 & 81 & 59 & 100 & 82 & 92 & 63 & 59 & 62 & 87 & 52 & 115 & 78 & 110 & 69 & 93 & 77 & 65 & 53 \\
\hline $\begin{array}{l}\text { Marmalade, jam and } \\
\text { sugar }\end{array}$ & 25 & 21 & 21 & 18 & 21 & 19 & 32 & 24 & 28 & 25 & 22 & 19 & 25 & 21 & $14 \dagger$ & $12 \dagger$ & $20 \dagger$ & $14 \dagger$ & $35+$ & $26 \dagger$ & 28 & 25 & 23 & 20 & 23 & 18 \\
\hline Soft drink & 44 & 94 & 29 & 83 & 45 & 101 & 59 & 9 & 54 & 97 & 41 & 85 & 39 & 101 & 28 & 65 & 45 & 93 & 53 & 109 & 46 & 97 & 40 & 86 & 47 & 100 \\
\hline Alcoh & 153 & 143 & $236+$ & $167 \dagger$ & $117 \dagger$ & $97 \dagger$ & $91 \dagger$ & $94 \dagger$ & 171 & 167 & 166 & 143 & 126 & 119 & 116 & 118 & 166 & 160 & 167 & 142 & 125 & 131 & 159 & 138 & 185 & 158 \\
\hline Alcohol content $>4 \%$ & 33 & 55 & $65 \dagger$ & $72 \dagger$ & $19 \dagger$ & $26+$ & $10 \dagger$ & $22 \dagger$ & 32 & 58 & 34 & 47 & 34 & 59 & 33 & 45 & 35 & 59 & 33 & 57 & 33 & 44 & 36 & 66 & 31 & 54 \\
\hline
\end{tabular}

Values are presented as mean daily intake in grams (sD).

"Food groups are defined and exempl

$\dagger$ Food groups with principal components loadings $>0.30$ and $<-0.30$. 
Table 2. Characteristics at baseline (mean age 71) and follow-up (mean age 87) of men included in the main analysis and grouped according to low, respectively, high adherence to each dietary pattern at baseline

(Mean values and standard deviations; numbers and percentages).

\begin{tabular}{|c|c|c|c|c|c|c|c|c|c|c|c|c|c|c|c|c|c|c|c|}
\hline & & & & \multicolumn{4}{|c|}{ DP 1} & \multicolumn{4}{|c|}{ DP 2} & \multicolumn{4}{|c|}{ DP 3} & \multicolumn{4}{|c|}{ DP 4} \\
\hline & & \multicolumn{2}{|c|}{ Total ( $n$ 257) } & \multicolumn{2}{|c|}{ Low ( $n 98)$} & \multicolumn{2}{|c|}{ High ( $n$ 91) } & \multicolumn{2}{|c|}{ Low $(n 74)$} & \multicolumn{2}{|c|}{ High ( $n$ 92) } & \multicolumn{2}{|c|}{ Low $(n 67)$} & \multicolumn{2}{|c|}{ High ( $n$ 109) } & \multicolumn{2}{|c|}{ Low $(n 100)$} & \multicolumn{2}{|c|}{ High ( $n 74)$} \\
\hline & & Mean & SD & Mean & SD & Mean & SD & Mean & SD & Mean & SD & Mean & SD & Mean & SD & Mean & SD & Mean & SD \\
\hline Age (years) & baseline & 70.9 & 0.6 & 71.0 & 0.6 & 71.0 & 0.6 & 71.0 & 0.6 & 70.9 & 0.6 & 70.9 & 0.6 & 71.0 & 0.6 & 70.9 & 0.6 & 70.9 & 0.7 \\
\hline $\begin{array}{l}\text { Follow-up period } \\
\text { (years) }\end{array}$ & & $15 \cdot 7$ & 0.7 & $15 \cdot 6$ & 0.7 & $15 \cdot 6$ & 0.7 & $15 \cdot 7$ & 0.7 & $15 \cdot 6$ & 0.7 & $15 \cdot 6$ & 0.6 & $15 \cdot 7$ & 0.7 & $15 \cdot 6$ & 0.7 & $15 \cdot 6$ & 0.6 \\
\hline Body weight (kg) & baseline & 79.5 & 9.9 & 80.6 & 9.9 & 77.8 & 10.9 & 80.0 & 9.6 & 78.9 & 8.7 & 80.6 & 12.5 & $78 \cdot 4$ & 8.9 & 80.0 & 10.6 & 79.5 & 9.4 \\
\hline Body weight (kg) & follow-up & $76 \cdot 2$ & 11.5 & 76.5 & 11.0 & 74.7 & $13 \cdot 2$ & 76.0 & 13.3 & 76.4 & $10 \cdot 1$ & $75 \cdot 7$ & 13.0 & 76.0 & $10 \cdot 1$ & 76.9 & 12.5 & $76 \cdot 1$ & 10.6 \\
\hline $\mathrm{BMI}\left(\mathrm{kg} / \mathrm{m}^{2}\right)$ & baseline & $25 \cdot 9$ & 2.9 & $26 \cdot 0$ & 2.9 & $25 \cdot 4$ & 3.0 & $26 \cdot 1$ & 3.0 & $25 \cdot 6$ & 2.6 & $26 \cdot 2$ & 3.5 & $25 \cdot 4$ & $2 \cdot 3$ & $25 \cdot 9$ & 3.0 & $25 \cdot 9$ & $2 \cdot 8$ \\
\hline BMI $\left(\mathrm{kg} / \mathrm{m}^{2}\right)$ & follow-up & $25 \cdot 6$ & 3.5 & $25 \cdot 5$ & 3.3 & $25 \cdot 2$ & 4.0 & $25 \cdot 7$ & 4.4 & 25.5 & 3.1 & 25.4 & 3.9 & $25 \cdot 3$ & $2 \cdot 9$ & $25 \cdot 7$ & 3.7 & $25 \cdot 6$ & 3.4 \\
\hline $\begin{array}{l}\text { Appendicular LMI (kg/ } \\
\left.\mathrm{m}^{2}\right)\end{array}$ & follow-up & 7.5 & 0.8 & 7.4 & 0.8 & 7.5 & 0.8 & 7.4 & 0.9 & 7.5 & 0.6 & $7 \cdot 4$ & 0.8 & 7.5 & 0.7 & $7 \cdot 5$ & 0.8 & 7.4 & 0.7 \\
\hline $\begin{array}{l}\text { Reported energy intake } \\
(\mathrm{kcal} / \mathrm{d})\end{array}$ & baseline & 1853 & 441 & 1700 & 387 & 2018 & 459 & 1744 & 423 & 1972 & 456 & 1407 & 248 & 2152 & 376 & 1813 & 407 & 1959 & 470 \\
\hline $\begin{array}{l}\text { Reported protein intake } \\
\text { (g/d) }\end{array}$ & baseline & 69 & 16 & 63 & 13 & 74 & 17 & 64 & 14 & 74 & 18 & 55 & 10 & 79 & 15 & 65 & 14 & 76 & 18 \\
\hline $\begin{array}{l}\text { Reported protein intake } \\
\text { ( } \mathrm{g} / \mathrm{kg} \text { body weight) }\end{array}$ & baseline & 0.88 & 0.24 & 0.80 & 0.20 & 0.97 & 0.27 & 0.81 & 0.20 & 0.95 & 0.25 & 0.70 & 0.18 & 1.02 & 0.23 & 0.83 & 0.19 & 0.98 & 0.27 \\
\hline Years of education $(n)$ & & $n$ & $\%$ & $n$ & $\%$ & $n$ & $\%$ & $n$ & $\%$ & $n$ & $\%$ & $n$ & $\%$ & $n$ & $\%$ & $n$ & $\%$ & $n$ & $\%$ \\
\hline $6-7$ years & & 122 & 47 & 29 & 30 & 56 & 62 & 37 & 50 & 36 & 39 & 37 & 55 & 45 & 41 & 39 & 39 & 44 & 59 \\
\hline $8-13$ years & & 81 & 32 & 34 & 35 & 26 & 29 & 24 & 32 & 30 & 33 & 16 & 24 & 40 & 37 & 33 & 33 & 21 & 28 \\
\hline$>13$ years & & 54 & 21 & 35 & 36 & 9 & 10 & 13 & 18 & 26 & 28 & 14 & 21 & 24 & 22 & 28 & 28 & 9 & 12 \\
\hline $\begin{array}{l}\text { Physical activity level } \\
(n)\end{array}$ & baseline & & & & & & & & & & & & & & & & & & \\
\hline Low & & 81 & 32 & 34 & 35 & 30 & 33 & 20 & 27 & 28 & 30 & 27 & 40 & 33 & 30 & 32 & 32 & 19 & 26 \\
\hline Medium & & 156 & 61 & 54 & 55 & 58 & 64 & 51 & 69 & 56 & 61 & 37 & 55 & 67 & 61 & 57 & 57 & 52 & 70 \\
\hline High & & 20 & 8 & 10 & 10 & 3 & 3 & 3 & 4 & 8 & 9 & 3 & 4 & 9 & 8 & 11 & 11 & 3 & 4 \\
\hline Smoking $(n)$ & baseline & & & & & & & & & & & & & & & & & & \\
\hline Never & & 104 & 40 & 41 & 42 & 38 & 42 & 29 & 39 & 38 & 41 & 15 & 22 & 49 & 54 & 43 & 43 & 26 & 35 \\
\hline Current & & 28 & 11 & 11 & 11 & 11 & 12 & 8 & 11 & 6 & 7 & 17 & 25 & 5 & 5 & 11 & 11 & 8 & 11 \\
\hline Former & & 125 & 49 & 46 & 47 & 42 & 46 & 37 & 50 & 48 & 52 & 35 & 52 & 55 & 50 & 46 & 46 & 40 & 54 \\
\hline $\begin{array}{l}\text { Charlson Comorbidity } \\
\text { Index (n) }\end{array}$ & baseline & & & & & & & & & & & & & & & & & & \\
\hline 0 & & 202 & 79 & 77 & 79 & 73 & 80 & 60 & 81 & 72 & 78 & 52 & 78 & 86 & 79 & 77 & 77 & 64 & 86 \\
\hline$\geq 1$ & & 55 & 21 & 21 & 21 & 18 & 20 & 14 & 19 & 20 & 22 & 15 & 22 & 23 & 21 & 23 & 23 & 10 & 14 \\
\hline
\end{tabular}

Values are presented as mean (standard deviation) for continuous measures and number (percentage) for categorical measures.

LMI, lean mass index. 
Table 3. Logistic regression analysis between adherence to each dietary pattern at baseline (mean age 71) and prevalence of sarcopenia defined according to EWGSOP2 at follow-up (mean age 87)

(Odd ratio and $95 \%$ confidence intervals).

\begin{tabular}{|c|c|c|c|c|c|c|c|}
\hline & \multicolumn{7}{|c|}{ Adherence to dietary pattern } \\
\hline & \multirow{2}{*}{$\frac{\text { Low }}{\text { OR }}$} & \multicolumn{2}{|c|}{ Medium } & \multicolumn{2}{|c|}{ High } & \multicolumn{2}{|c|}{$\begin{array}{l}\text { Continuous (per } 1 \mathrm{SD} \text { ) } \\
\text { increment) }\end{array}$} \\
\hline & & OR & $95 \% \mathrm{Cl}$ & OR & $95 \% \mathrm{Cl}$ & OR & $95 \% \mathrm{Cl}$ \\
\hline \multicolumn{8}{|l|}{ DP 1} \\
\hline $\begin{array}{l}\text { Participants, } n \\
\text { Sarcopenia }\end{array}$ & 98 & 68 & & 91 & & 257 & \\
\hline$n$ & 17 & 13 & & 20 & & 50 & \\
\hline$\%$ & 17 & 19 & & 22 & & 19 & \\
\hline Model $1^{*}$ & 1.00 (ref) & $1 \cdot 13$ & $0.51,2.50$ & 1.34 & $0.65,2.76$ & $1 \cdot 11$ & $0.85,1.46$ \\
\hline Model $2 \dagger$ & 1.00 (ref) & $1 \cdot 12$ & $0.48,2.60$ & $1 \cdot 34$ & $0.59,3.02$ & $1 \cdot 13$ & $0.82,1.56$ \\
\hline $\begin{array}{l}\text { Model } 3 \ddagger \\
\text { DP } 2\end{array}$ & 1.00 (ref) & $1 \cdot 22$ & $0.49,3.02$ & $1 \cdot 13$ & $0.48,2 \cdot 70$ & 1.04 & $0.74,1.46$ \\
\hline $\begin{array}{l}\text { Participants, } n \\
\text { Sarcopenia }\end{array}$ & 74 & 91 & & 92 & & 257 & \\
\hline$n$ & 20 & 15 & & 15 & & 50 & \\
\hline$\%$ & 27 & 16 & & 16 & & 19 & \\
\hline Model $1^{\star}$ & 1.00 (ref) & 0.53 & $0.25,1.13$ & 0.53 & $0.25,1.12$ & 0.79 & $0.57,1.11$ \\
\hline Model 2† & 1.00 (ref) & 0.49 & $0.23,1.08$ & 0.48 & $0.22,1.07$ & 0.76 & $0.53,1.07$ \\
\hline Model 3‡ & 1.00 (ref) & 0.41 & $0.17,0.98$ & 0.40 & $0.17,0.94$ & 0.70 & $0.48,1.03$ \\
\hline DP 3 & & & & & & & \\
\hline $\begin{array}{l}\text { Participants, } n \\
\text { Sarcopenia }\end{array}$ & 67 & 81 & & 109 & & 257 & \\
\hline$n$ & 17 & 12 & & 21 & & 50 & \\
\hline$\%$ & 25 & 15 & & 19 & & 19 & \\
\hline Model 1* & 1.00 (ref) & 0.51 & $0.22,1.17$ & 0.70 & $0.34,1.45$ & 1.06 & $0.79,1.42$ \\
\hline Model 2† & 1.00 (ref) & 0.47 & $0.18,1.24$ & $0 \cdot 70$ & $0.21,1.81$ & 1.41 & $0.85,2.32$ \\
\hline $\begin{array}{l}\text { Model 3ł } \\
\text { DP } 4\end{array}$ & 1.00 (ref) & 0.50 & $0.18,1.40$ & 0.53 & $0.17,1.70$ & $1 \cdot 19$ & $0.71,2.00$ \\
\hline $\begin{array}{l}\text { Participants, } n \\
\text { Sarcopenia }\end{array}$ & 100 & 83 & & 74 & & 257 & \\
\hline$n$ & 16 & 18 & & 16 & & 50 & \\
\hline$\%$ & 16 & 22 & & 22 & & 19 & \\
\hline Model $1^{*}$ & 1.00 (ref) & 1.45 & $0.69,3.07$ & 1.45 & $0.67,3.13$ & 1.04 & $0.76,1.41$ \\
\hline Model 2† & 1.00 (ref) & 1.53 & $0.71,3.31$ & 1.47 & $0.66,3.30$ & 1.05 & $0.76,1.47$ \\
\hline Model 3† & 1.00 (ref) & 1.57 & $0.69,3.57$ & 1.61 & $0.67,3.87$ & 1.08 & $0.77,1.53$ \\
\hline
\end{tabular}

$\mathrm{DP}$, dietary pattern.

Participants were categorised as low, medium or high adherent to each DP based on factor scores and according to tertiles limits determined based on adherence to dietary patterns at baseline in the total population of 1133 men.

${ }^{*}$ Model 1: unadjusted for potential confounders.

† Model 2: adjusted for age at baseline (continuous), follow-up period (continuous), reported energy intake at baseline (continuous), education (categorical), physical activity at base-

line (categorical), smoking (categorical) and morbidity at baseline (categorical).

¥ Model 3: further adjusted for BMI at baseline (continuous).

\section{Dietary patterns and the variables used in the definition} of sarcopenia

The analyses of DP in relation to the individual variables used in the definition of sarcopenia displayed no clear associations (Fig. 1 and online Supplementary Table 8). However, higher adherence to DP3 was associated with higher handgrip strength, which was statistically significant if unadjusted, but not if adjusted according to model 2 or 3. Medium adherence to DP4 was associated with lower handgrip strength and slower gait speed, which was statistically significant both unadjusted and adjusted. Medium adherence to DP4 was also associated with longer time to perform chair stand test, which was statistically significant if unadjusted, but not if adjusted according to model 2 or 3.

\section{Sensitivity analyses}

In a subgroup analysis ( $n$ 247), we excluded participants ( $n$ 10) with self-reported body weight loss in the past year before the baseline measurement to limit potential influences of recent change of dietary intake due to, for example, undiagnosed disease before baseline. This exclusion did not affect the overall associations between the DP and sarcopenia (EWGSOP2) (online Supplementary Table 9).

In another subgroup analysis ( $n$ 237), we excluded participants with BMI below 22 at baseline ( $n$ 20) to limit the potential risk of participants having low muscle mass at baseline. This exclusion did not significantly affect the association between DP and sarcopenia (EWGSOP2), with the exception of wider CI (online Supplementary Table 10). 
(A)

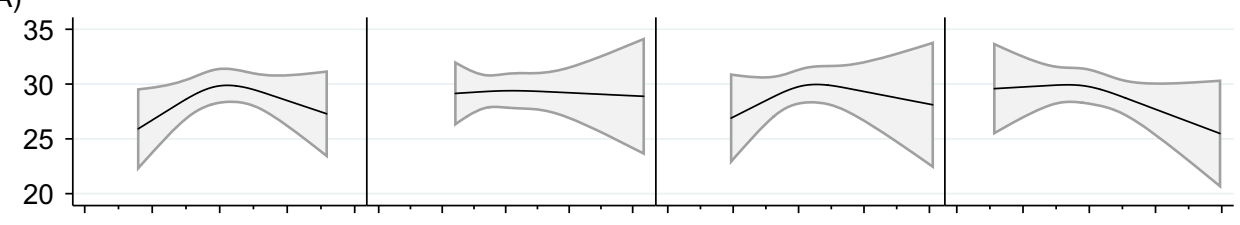

(B)

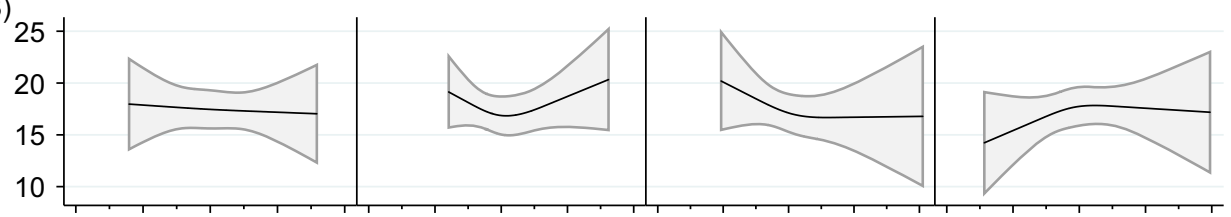

(C)

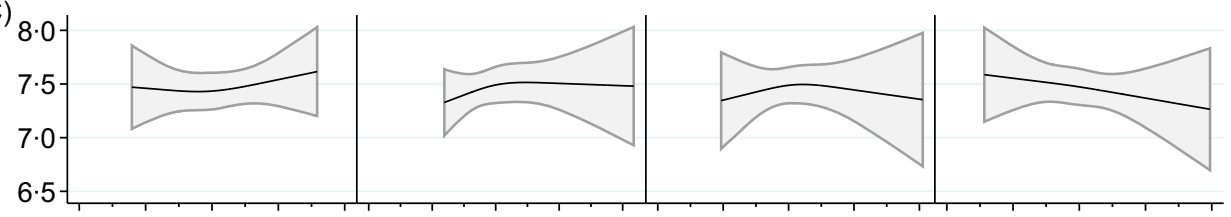

(D)

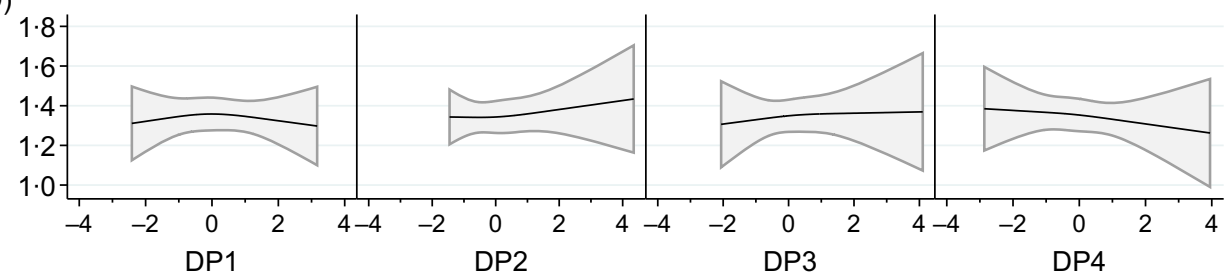

Fig. 1. Associations between dietary pattern (DP) 1-4 with the variables used in the definition of sarcopenia: A. hand grip strength (kg), $n 301, \mathrm{~B}$. chair stand test (seconds), $n 241$, C. appendicular lean mass index $\left(\mathrm{kg} / \mathrm{m}^{2}\right), n 257$ and D. gait speed $(\mathrm{m} / \mathrm{s}), n 257$. Beta estimates (on each $\mathrm{Y}$ axis) were modelled using restricted cubic splines (three knots placed at the 10th, 50th and 90th percentiles) and were adjusted for age at baseline (continuous), follow-up period (continuous), reported energy intake at baseline (continuous), education (categorical), physical activity at baseline (categorical), smoking (categorical), morbidity at baseline (categorical) and BMI at baseline (continuous), i.e. Model 3 . The solid line represents the beta coefficient and the shaded area represents its $95 \% \mathrm{Cl}$.

In addition, we derived DP, using the same method and thresholds as in the main study group ( $n$ 1133), in a subgroup of adequate energy reporters ( $n$ 855), according to the Goldberg cut-offs ${ }^{(52)}$, based on individual specific energy requirements. The derived DP and the associations between the DP and sarcopenia (EWGSOP2) were largely similar to our main analysis, however, with larger CI (online Supplementary Material 2).

\section{Discussion}

In this cohort of 71-year-old Swedish men, adherence to a dietary pattern mainly characterised by a high consumption of fruit, vegetables, poultry, rice and pasta (DP2) was associated with lower prevalence of sarcopenia defined according to EWGSOP2, 16 years later. In contrast, a higher adherence to a dietary pattern mainly characterised by a high consumption of potato, meat and egg and low consumption of fermented milk (DP4) was associated with higher prevalence of sarcopenia, although with low precision. These results add potentially important knowledge to the limited literature using dietary patterns rather than nutrients or single foods, to investigate the association between dietary intake and sarcopenia.

Methods used when defining DP are often subdivided into a priori and a posteriori. Several a priori $\mathrm{DP}$ are linked to dietary guidelines and thus based on the current state of knowledge, with the limitation that they do not necessarily capture the complexity of the total diet. A posteriori defined DP are, in contrast, statistically derived patterns from the dietary data at hand without prior hypotheses about the association between diet and outcome and are to a larger extent population specific. A posteriori approaches can, thus, be useful when knowledge of the association between diet and outcome is limited, as with diet and sarcopenia. Factor analysis, including PCA used in the present study, calculates a score for each individual's adherence to each DP, reflecting that most participants eat all types of foods, albeit in different amounts and frequencies. In cluster analysis, each participant contributes to one single derived cluster. Given that, from a statistical point of view, our study population is small, factor analysis may be preferable.

In PCA-derived DP, all included food groups contribute to each DP and explain the total diet; low or no intake of foods may be as important as foods consumed in larger amounts. We, therefore, label the DP in our study as DP1, DP2, DP3 and DP4, rather than label them as 'Western' or 'Healthy'. As none of the previously published studies includes DP similar to DP1 or DP3, and we do not display any clear associations, these are not discussed further. The DP explained $28 \%$ of the total variance, leaving $72 \%$ of the variance unexplained. This is similar to what has been reported from other studies and may be considered as fairly high explanation rate due to the diversity of dietary habits within a population. 
Previous longitudinal studies are few and reported no associations between a posteriori DP and incident sarcopenia in community-dwelling men and women in Hong Kong ${ }^{(24)}$ (age $\geq$ 65 years, 4-year follow-up) and $\mathrm{UK}^{(53)}$ (age $\geq 85$ years; 3-year follow-up). However, cross-sectional associations were observed in these, and other, studies. A DP characterised by a high consumption of butter, red meat, gravy, vegetables, sweets/dessert and potatoes was associated with higher prevalence of sarcopenia ${ }^{(53)}$. DP characterised by a high intake of poultry ${ }^{(54)}$; vegetables, fruit, fish, nuts and vegetable oil ${ }^{(25,26)}$; vegetables and fruits ${ }^{(24)}$; snacks, coffee, milk and fast food ${ }^{(24)}$; vegetables, fruit, fish and tofu ${ }^{(55)}$ were all associated with a lower prevalence of sarcopenia. Even though most of previous studies report that high adherence to DP characterised by high intake of vegetables and fruit is associated with lower prevalence of sarcopenia, the total diet seems important, and a high intake of vegetables alone may not be enough as dissonant results have been reported $^{(23,24,53)}$. Dietary patterns add context to the single food items. Dissimilar findings may be explained by regional or cultural differences in how foods are used in cooking or combined in meals, how foods are combined into food groups by the researchers, the definition of sarcopenia (e.g. EWGSOP1 vs. EWGSOP2), and follow-up period.

Based on the same cohort as in this study, we displayed that a Mediterranean-like diet was associated with lower prevalence of sarcopenia (EWGSOP1) ${ }^{(23)}$. Despite the similarities of the Mediterranean diet with DP2 in the present study, we did not see an association of DP2 with sarcopenia according to EWGSOP1 in the present study. Although several aspects are similar between DP2 in the current study and the Mediterranean-like diet used in our previous study ${ }^{(23)}$, the factor loading of alcohol in DP2 is low but is included as one item in the Mediterranean-like diet. However, the significance of this is difficult to estimate. The sarcopenia definitions partly capture different individuals and differ mainly in the decisive variables used; the chair stand test in EWGSOP2 (main outcome in the present study) and handgrip strength in EWGSOP1. In the present study, DP2 was associated with sarcopenia defined as the combination of muscle function and muscle mass, but not with the components of sarcopenia. This could be an indication that the combination of an impaired physical function, low muscle mass and poor strength may be more detrimental than each component on its own, similar to the complexity of dietary intake where DP represent a more complete picture than single foods or nutrients. However, a high adherence to DP 4 seemed to have an inverse association with muscle function and muscle mass. A similar DP characterised by, among other food groups, high intake of red meat and potatoes was inversely associated with muscle strength and physical performance ${ }^{(53)}$.

In the present study, DP were calculated based on the actual intake of each food group in grams ${ }^{(21)}$ and may represent different energy densities. To ensure that any associations between the DP and the outcome are not due to differences in energy intake, we include energy intake as a covariate in the multivariable analyses and excluded extreme outliers of energy intake. This allows for ranking of individuals' dietary intakes, which is essential when studying associations ${ }^{(28)}$. Additional exclusion of inadequate energy reporters ${ }^{(52)}$ in a sensitivity analysis is described in Supplementary Material 2. To limit misreporting of dietary intake, we used a valid 7-d menu book (online Supplementary Material 1) that was filled in prospectively and allowed for adequate calculation of total energy intake, however, with potential selection of what is eaten during this specific period. An FFQ would cover a larger period and might provide a broader picture but is on the other hand recorded retrospectively.

Several studies, examining single food groups, have reported a high intake of fruit and vegetables being associated with lower prevalence of sarcopenia ${ }^{(56)}$, in line with our and previous studies on dietary patterns. Mechanisms may involve anti-oxidative ${ }^{(57)}$ and anti-inflammatory ${ }^{(58)}$ effects on skeletal muscle and ageing, potentially involving the phytochemical content of fruits and vegetables $^{(56)}$ or the acid-base balance of the diet, as net acid load has been reported to be associated with loss of muscle mass ${ }^{(59)}$. A high intake of protein has been associated with muscle mass ${ }^{(60)}$, and a higher intake of protein per $\mathrm{kg} /$ body weight has been recommended $^{(61)}$. In this context, it is interesting to note that a higher adherence to our DP2 was associated with a higher absolute protein intake (online Supplementary Table 6). However, Granic et al. have reported that a DP (high in butter, red meat, gravy and potato) was associated with an increased risk of sarcopenia, even when the intake of protein was high ${ }^{(53)}$.

Strengths of the present study include the longitudinal approach with a long follow-up period, a well-characterised population, and that we adjust for a large number of potential confounders including BMI and physical activity that are associated with both energy requirement and lifestyle. The relatively small study population was partly compensated for by an age-standardised set-up and a follow-up period well adapted to the period when sarcopenia begins to become more common ${ }^{(62-65)}$. As the age-related decline of muscle mass and function is slow, and the sarcopenia definition is based on thresholds, a long followup period is crucial. We created the DP among the full population at baseline, and not only among those attending the follow-up, to better reflect the distribution of the DP at that time. However, it should be borne in mind that the diet among 71-year-old men in the 1990s probably does not correspond to a typical diet in that age group in the 2020s. Dietary patterns are influenced by cultural eating habits and may therefore not be easily transferable to other populations $^{(21)}$. This is somewhat counteracted by the fact that we are ranking individuals according to DP adherence and do not focus on specific amounts of foods or nutrients.

Using a single measure of dietary intake as a reflection of habitual dietary habits may be seen as a limitation as the dietary intake may change over time although adherence to dietary patterns has been reported to be reasonably stable during 10 years in Swedish and other populations ${ }^{(66-68)}$. However, if dietary habits changed during the follow-up period, it would probably affect our estimates towards the null.

The main limitation of the present study is that we were not able to define sarcopenia at baseline. The prevalence of sarcopenia at an approximate age of 70 in this population is usually considered as low ${ }^{(64)}$. We did not see a major impact on our estimates by exclusion of those with a low BMI at baseline, being at higher risk of having sarcopenia. Nonetheless, we cannot preclude the possibility of reverse causation. There may be an influence of 
residual or unmeasured confounding, and we cannot exclude that any statistically significant associations may have occurred by chance. Because sarcopenia was defined on average 16 years after baseline at a follow-up examination, participants included in our study had a higher physical activity level and fewer comorbidities at baseline, compared to excluded participants, a possible result of a healthy survivor effect or natural selection by increasing age, introducing potential selection bias. However, since many confounders in our adjusted model are linked with reasons for nonparticipation, the influence of such selection bias is limited ${ }^{(69)}$. The participants in our study probably had a normal nutritional status at baseline, even though we lack specific information on nutritional status. Our results may not be generalisable to a more sedentary population, to a population with more comorbidities or with great cultural differences in eating habits, to other age groups, or to women ${ }^{(17,63-65)}$.

\section{Conclusions}

In summary, using an a posteriori approach and reported dietary intake in a cohort of community-dwelling 71-year-old Swedish men, we defined four DP. A higher adherence to DP2, mainly characterised by high consumption of vegetables, green salad and fruit as well as poultry, rice and pasta, was associated with a lower prevalence of sarcopenia defined according to EWGSOP2 16 years later. Contradicting results when comparing studies, using different methods, stress the need to use equivalent definitions and methodology when defining sarcopenia. Thus, it could be of value to consider reanalysis of some previous studies, using EWGSOP1 and EWGSOP2 for the diagnosis of sarcopenia.

\section{Acknowledgements}

We acknowledge the contribution of Käthe Ström, research nurse and Vilmantas Giedraitis, ULSAM data manager. We acknowledge Inga-Britt Gustafsson and Lars Berglund (Department of Public Health and Caring Sciences, Uppsala University) for the work they invested in the development of the menu book.

The Uppsala Geriatric Foundation and the Region Örebro County supported this work. These funding sources had no role in the design, conduct of research or decision of publication.

Conceptualisation, M. K., W. B., T. C. and L. B.; methodology, M. K., W. B., T. C. and L. B.; formal analysis, M. K.; data curation, M. K.; writing - original draft preparation, M. K.; writing - review and editing, M. K., W. B., T. C. and L. B.; supervision, W. B., T. C. and L. B. All authors have read and agreed to the published version of the manuscript.

None. The authors declare no conflict of interest. The funders had no role in the design of the study; in the collection, analyses or interpretation of data; in the writing of the manuscript or in the decision to publish the results.

\section{Supplementary material}

For supplementary material/s referred to in this article, please visit https://doi.org/10.1017/S0007114521003901

\section{References}

1. Rosenberg IH (1997) Sarcopenia: origins and clinical relevance. J Nutr 127, 990s-991s.

2. Roubenoff R (2000) Sarcopenia and its implications for the elderly. Eur J Clin Nutr 54, S40-S47.

3. Beaudart C, Biver E, Reginster JY, et al. (2017) Validation of the SarQoL(R), a specific health-related quality of life questionnaire for Sarcopenia. J Cachexia Sarcopenia Muscle 8, 238-244.

4. Beaudart C, Locquet M, Reginster JY, et al. (2018) Quality of life in sarcopenia measured with the SarQoL(R): impact of the use of different diagnosis definitions. Aging Clin Exp Res 30, 307-313.

5. Muscaritoli M, Anker SD, Argilés J, et al. (2010) Consensus definition of sarcopenia, cachexia and pre-cachexia: joint document elaborated by Special Interest Groups (SIG) 'cachexia-anorexia in chronic wasting diseases' and 'nutrition in geriatrics'. Clin Nutr 29, 154-159.

6. Fielding RA, Vellas B, Evans WJ, et al. (2011) Sarcopenia: an undiagnosed condition in older adults. Current consensus definition: prevalence, etiology, and consequences. International working group on sarcopenia. J Am Med Dir Assoc 12, 249-256.

7. Morley JE, Abbatecola AM, Argiles JM, et al. (2011) Sarcopenia with limited mobility: an international consensus. J Am Med Dir Assoc 12, 403-409.

8. Chen LK, Liu LK, Woo J, et al. (2014) Sarcopenia in Asia: consensus report of the Asian working group for sarcopenia. $J \mathrm{Am}$ Med Dir Assoc 15, 95-101.

9. Studenski SA, Peters KW, Alley DE, et al. (2014) The FNIH sarcopenia project: rationale, study description, conference recommendations, and final estimates. J Gerontol A Biol Sci Med Sci 69, 547-558.

10. Goodpaster BH, Park SW, Harris TB, et al. (2006) The loss of skeletal muscle strength, mass, and quality in older adults: the health, aging and body composition study. J Gerontol A Biol Sci Med Sci 61, 1059-1064.

11. Clark BC \& Manini TM (2008) Sarcopenia $=/=$ dynapenia. $J$ Gerontol A Biol Sci Med Sci 63, 829-834.

12. Delmonico MJ, Harris TB, Visser M, et al. (2009) Longitudinal study of muscle strength, quality, and adipose tissue infiltration. Am J Clin Nutr 90, 1579-1585.

13. Visser M \& Schaap LA (2011) Consequences of sarcopenia. Clin Geriatr Med 27, 387-399.

14. Cruz-Jentoft AJ \& Sayer AA (2019) Sarcopenia. Lancet 393 , 2636-2646

15. Denison HJ, Cooper C, Sayer AA, et al. (2015) Prevention and optimal management of sarcopenia: a review of combined exercise and nutrition interventions to improve muscle outcomes in older people. Clin Interv Aging 10, 859-869.

16. Beaudart C, Dawson A, Shaw SC, et al. (2017) Nutrition and physical activity in the prevention and treatment of sarcopenia: systematic review. Osteoporos Int 28, 1817-1833.

17. Bloom I, Shand C, Cooper C, et al. (2018) Diet quality and sarcopenia in older adults: a systematic review. Nutrients 10, 308.

18. Granic A, Sayer AA \& Robinson SM (2019) Dietary patterns, skeletal muscle health, and sarcopenia in older adults. Nutrients 11, 745 .

19. Jacobs DR, Gross MD \& Tapsell LC (2009) Food synergy: an operational concept for understanding nutrition. Am J Clin Nutr 89, 1543s-1548s.

20. Jacobs DR \& Tapsell LC (2007) Food, not nutrients, is the fundamental unit in nutrition. Nutr Rev 65, 439-450.

21. Hu FB (2002) Dietary pattern analysis: a new direction in nutritional epidemiology. Curr Opin Lipidol 13, 3-9.

22. Randall E, Marshall JR, Graham S, et al. (1990) Patterns in food use and their associations with nutrient intakes. Am J Clin Nutr 52, 739-745. 
23. Karlsson M, Becker W, Michaëlsson K, et al. (2020) Associations between dietary patterns at age 71 and the prevalence of sarcopenia 16 years later. Clin Nutr 39, 1077-1084.

24. Chan R, Leung J \& Woo J (2016) A prospective cohort study to examine the association between dietary patterns and sarcopenia in Chinese community-dwelling older people in Hong Kong. J Am Med Dir Assoc 17, 336-342.

25. Mohseni R, Aliakbar S, Abdollahi A, et al. (2017) Relationship between major dietary patterns and sarcopenia among menopausal women. Aging Clin Exp Res 29, 1241-1248.

26. Hashemi R, Motlagh AD, Heshmat R, et al. (2015) Diet and its relationship to sarcopenia in community dwelling Iranian elderly: a cross sectional study. Nutrition 31, 97-104.

27. Uppsala longitudinal study of adult men (ULSAM) (2020) Uppsala Longitudinal Study of Adult Men (ULSAM). http:// www.pubcare.uu.se/ulsam (accessed October 2020).

28. Willett W (2013) Nutritional Epidemiology, 3rd ed. Oxford: Oxford University Press.

29. Cruz-Jentoft AJ, Bahat G, Bauer J, et al. (2019) Sarcopenia: revised European consensus on definition and diagnosis. Age Ageing 48, 16-31.

30. Rosell M, Hellénius M-L, de Faire U, et al. (2003) Contribution of a manually coded part in an optically readable, precoded seven-day food record for the intake of energy, nutrients and foods. Scand J Nutr 47, 123-131.

31. Nydahl M, Gustafsson IB, Mohsen R, et al. (2009) Comparison between optical readable and open-ended weighed food records. Food Nutr Res 53, 1889.

32. Martiin C (2010) Swedish milk, a Swedish duty: dairy marketing in the 1920's and 1930's. Rural Hist 21, 213-232.

33. Scander H, Monteagudo C, Nilsen B, et al. (2018) Beverage consumption patterns and energy contribution from beverages per meal type: results from a national dietary survey in Sweden. Public Health Nutr 21, 3318-3327.

34. Northstone K, Ness AR, Emmett PM, et al. (2008) Adjusting for energy intake in dietary pattern investigations using principal components analysis. Eur J Clin Nutr 62, 931-938.

35. Varraso R, Garcia-Aymerich J, Monier F, et al. (2012) Assessment of dietary patterns in nutritional epidemiology: principal component analysis compared with confirmatory factor analysis. Am J Clin Nutr 96, 1079-1092.

36. Cruz-Jentoft AJ, Baeyens JP, Bauer JM, et al. (2010) Sarcopenia: European consensus on definition and diagnosis: report of the European Working Group on sarcopenia in older people. Age Ageing 39, 412-423.

37. Dodds RM, Syddall HE, Cooper R, et al. (2014) Grip strength across the life course: normative data from twelve British studies. PLoS One 9, e113637.

38. Cesari M, Kritchevsky SB, Newman AB, et al. (2009) Added value of physical performance measures in predicting adverse health-related events: results from the health, aging and body composition study. J Am Geriatr Soc 57, 251-259.

39. Gould H, Brennan SL, Kotowicz MA, et al. (2014) Total and appendicular lean mass reference ranges for Australian men and women: the Geelong osteoporosis study. Calcif Tissue Int 94, 363-372.

40. Baumgartner RN, Koehler KM, Gallagher D, et al. (1998) Epidemiology of sarcopenia among the elderly in New Mexico. Am J Epidemiol 147, 755-763.

41. Lauretani F, Russo CR, Bandinelli S, et al. (2003) Age-associated changes in skeletal muscles and their effect on mobility: an operational diagnosis of sarcopenia. J Appl Physiol 95, 18511860.

42. Mathiowetz V, Vizenor L \& Melander D (2000) Comparison of baseline instruments to the Jamar dynamometer and the B\&L engineering pinch gauge. Occup Ther J Res 20, 147-162.
43. Melhus H, Snellman G, Gedeborg R, et al. (2010) Plasma 25-hydroxyvitamin D levels and fracture risk in a community-based cohort of elderly men in Sweden. I Clin Endocrinol Metab 95, 2637-2645.

44. Vessby B, Tengblad S \& Lithell H (1994) Insulin sensitivity is related to the fatty acid composition of serum lipids and skeletal muscle phospholipids in 70-year-old men. Diabetologia 37, 1044-1050.

45. Byberg L, Zethelius B, McKeigue PM, et al. (2001) Changes in physical activity are associated with changes in metabolic cardiovascular risk factors. Diabetologia 44, 2134-2139.

46. Lochen ML \& Rasmussen K (1992) The Tromso study: physical fitness, self reported physical activity, and their relationship to other coronary risk factors. J Epidemiol Community Health 46, 103-107.

47. Quan H, Sundararajan V, Halfon P, et al. (2005) Coding algorithms for defining comorbidities in ICD-9-CM and ICD-10 administrative data. Med Care 43, 1130-1139.

48. Charlson ME, Pompei P, Ales KL, et al. (1987) A new method of classifying prognostic comorbidity in longitudinal studies: development and validation. J Chronic Dis 40, 373-383.

49. Harrell FE (2015) Regression Modeling Strategies: With Applications to Linear Models, Logistic and Ordinal Regression, and Survival Analysis. Cham: Springer.

50. Textor J, Hardt J \& Knüppel S (2011) DAGitty: a graphical tool for analyzing causal diagrams. Epidemiology 22, 745.

51. Hernán MA, Hernández-Díaz S, Werler MM, et al. (2002) Causal knowledge as a prerequisite for confounding evaluation: an application to birth defects epidemiology. Am J Epidemiol 155, 176-184.

52. Black AE (2000) Critical evaluation of energy intake using the Goldberg cut-off for energy intake: basal metabolic rate. A practical guide to its calculation, use and limitations. Int J Obes Relat Metab Disord 24, 1119-1130.

53. Granic A, Mendonça N, Sayer AA, et al. (2020) Effects of dietary patterns and low protein intake on sarcopenia risk in the very old: the Newcastle 85+ study. Clin Nutr 39, 166-173.

54. Fanelli Kuczmarski M, Mason MA, Beydoun MA, et al. (2013) Dietary patterns and sarcopenia in an urban African American and White population in the USA. J Nutr Gerontol Geriatr 32, 291-316.

55. Suthuvoravut U, Takahashi K, Murayama H, et al. (2020) Association between traditional Japanese Diet washoku and sarcopenia in community-dwelling older adults: findings from the Kashiwa study. J Nutr Health Aging 24, 282-289.

56. Granic A, Dismore L, Hurst C, et al. (2020) Myoprotective whole foods, muscle health and sarcopenia: a systematic review of observational and intervention studies in older adults. Nutrients 12, 2257.

57. Dobrowolny G, Barbiera A, Sica G, et al. (2021) Age-related alterations at neuromuscular junction: role of oxidative stress and epigenetic modifications. Cells 10, 1307.

58. Pascual-Fernández J, Fernández-Montero A, Córdova-Martínez A, et al. (2020) Sarcopenia: molecular pathways and potential targets for intervention. Int J Mol Sci 21, 8844.

59. Cruz-Jentoft AJ, Dawson Hughes B, Scott D, et al. (2020) Nutritional strategies for maintaining muscle mass and strength from middle age to later life: a narrative review. Maturitas $\mathbf{1 3 2}$, 57-64.

60. Houston DK, Nicklas BJ, Ding J, et al. (2008) Dietary protein intake is associated with lean mass change in older, community-dwelling adults: the Health, Aging, and Body Composition (Health ABC) Study. Am J Clin Nutr 87, 150-155.

61. Bauer J, Biolo G, Cederholm T, et al. (2013) Evidence-based recommendations for optimal dietary protein intake in older people: a position paper from the PROT-AGE Study Group. $J$ Am Med Dir Assoc 14, 542-559. 
62. Guillamón-Escudero C, Diago-Galmés A, Tenías-Burillo JM, et al. (2020) Prevalence of sarcopenia in CommunityDwelling Older Adults in Valencia, Spain. Int J Environ Res Public Health 17, 9130.

63. Yamada M, Nishiguchi S, Fukutani N, et al. (2013) Prevalence of sarcopenia in community-dwelling Japanese older adults. J Am Med Dir Assoc 14, 911-915.

64. Volpato S, Bianchi L, Cherubini A, et al. (2014) Prevalence and clinical correlates of sarcopenia in community-dwelling older people: application of the EWGSOP definition and diagnostic algorithm. J Gerontol A Biol Sci Med Sci 69, 438-446.

65. Akune T, Muraki S, Oka H, et al. (2014) Exercise habits during middle age are associated with lower prevalence of sarcopenia: the ROAD study. Osteoporos Int 25, 1081-1088.
66. Winkvist A, Klingberg S, Nilsson LM, et al. (2017) Longitudinal 10 -year changes in dietary intake and associations with cardiometabolic risk factors in the Northern Sweden Health and Disease Study. Nutr J 16, 20.

67. Keys A (1980) Seven Countries. A Multivariate Analysis of Death and Coronary Heart Disease. Cambridge: Harvard University Press.

68. Kimokoti RW, Newby PK, Gona P, et al. (2012) Stability of the Framingham nutritional risk score and its component nutrients over 8 years: the Framingham nutrition studies. Eur J Clin Nutr 66, 336-344

69. Nohr EA \& Liew Z (2018) How to investigate and adjust for selection bias in cohort studies. Acta Obstet Gynecol Scand 97, 407-416. 